\title{
Pseudo-Multiple-Exposure-based Tone Fusion and Visual-Salience-based Tone Mapping for High Dynamic Range Images: A Review
}

\author{
Sadaf Afreen \\ M. Tech. Scholar \\ All Saints College of \\ technology, \\ Bhopal M.P. India
}

\author{
Aizaz Tirmizi \\ Assistant Professor \\ All Saints College of \\ technology, \\ Bhopal M.P. India
}

\author{
Mohd. Sarwar Raeen \\ Head of Department - ECE \\ All Saints College of \\ technology, \\ Bhopal M.P. India
}

\begin{abstract}
This paper presents an introduction to novel saliency-aware weighting and edge-aware weighting for HDR images. These techniques are incorporated into an existing guided image filter to form a perceptually guided image filter. The saliencyaware weighting and the new filter are applied to design a new local tone-mapping algorithm for HDR images such that both extreme light and shadow regions can be reproduced on conventional low dynamic range displays. The VisualSalience-Based Tone Mapping (VSBTM) filter preserves sharp edges in the base layer better than the existing guided filter. Halo artifacts are thus significantly reduced in the tonemapped image. Moreover, the visual quality of the tonemapped image, especially attention-salient regions, is improved by the saliency-aware weighting. Pseudo-MultipleExposure-Based Tone Fusion (PMEBTF) gives a regionbased enhancement on the pseudo-exposures to boost details in the most distinct region. Thereby the region-enhanced pseudo-exposures are fused into an HDR image. The fused image thus enhances details in the bright region of the dark image and the dark region of the bright image.
\end{abstract}

\section{Keywords}

Edge-aware weighting, high dynamic range (HDR), local filtering, saliency-aware weighting, tone mapping.

\section{INTRODUCTION}

A conventional low dynamic range (LDR) image represents a scene at an exposure level with a limited contrast range. This results in loss of details in bright or dark areas of the scene depending on the setting of exposure level. A high dynamic range (HDR) image overcomes the limitation of the LDR image, and it can preserve details in both the bright and dark areas of the scene well [1]. Therefore, an HDR image includes much more information than an LDR image. However, the display of an HDR image is an issue. A global tone-mapping algorithm is simple and preserves naturalness of an HDR image because a global tone mapping algorithm compresses an HDR image using a monotonic curve [3]. However, the global tone-mapping algorithms face a fundamental difficulty in preserving the fine details of an HDR image [4]. It is thus desired to design a new local filter that can preserve sharp edges better so as to avoid halo artifacts from appearing in final images. In addition, visual saliency provides a mechanism for prioritizing visual processing, and it is shown to be useful for the processing of the conventional LDR images [2], [7], and [8]. It could be expected that visual saliency, like other perceptual quality measures such as perceived local contrast and color saturation in [11], is useful for HDR images. Unfortunately, it is not well utilized in the existing local tone-mapping algorithms. It is therefore also desired to build up a local tone-mapping algorithm by taking visual saliency into consideration. In VSBTM, a saliencyaware local tone-mapping algorithm is introduced for HDR images. Among the existing saliency models in [7]-[10], the saliency model in [9] and [10] is chosen to be extended from LDR domain to HDR domain due to its simplicity and robustness. The extended saliency model is adopted to set up a saliency-aware weighting for the processing of HDR images. In VSBTM the saliency-aware weighting and a new edge-aware weighting are fused together to build up a content-aware weighting which is incorporated into the guided image filter in [15] to form a perceptually guided image filter. The new filter and the saliency-aware weighting are then applied to design a local mapping algorithm for HDR images [14]. It was shown in [13] [14] that Reinhard's photographic tone reproduction [12] is one of the best local tone-mapping algorithms. Overall, the three major contributions of this VSBTM are the following: 1) a simple saliency-aware weighting for HDR images; 2) a contentaware guided image filter; and 3) a seamless integration of the, extend saliency-aware weighting and the global tonemapping algorithms in [12]. On the other side the inverse tone mapping method descried as inverse tone mapping operators (iTMOs) that work on reproducing real-world appearance images through LDR images [15]. It is done with a single camera; [HDR]_x simultaneously shoots two image tracks of whatever resolution and frame rate chosen.

During recording, there is no gap in time between the two separate image tracks to avoid an undesirable motion artifact. However, the major problem of such techniques is the high cost of the overall system since multiple sophisticated detectors or dedicated HDR sensors are required at the same time. Despite the increasing availability of HDR content, legacy LDR images and videos are still the majority [15]. Inverse tone mapping operators (iTMOs) that work on reproducing real-world appearance images through LDR images become more and more important recently [15]. The inverse tone mapping algorithms usually stretch the luminance range, adjust image contrast, and fill-in clipped (saturated) regions [18]. Region-based enhancement has been explored at iTMOs by identifying different parts in an image to adjust the content [17]. 


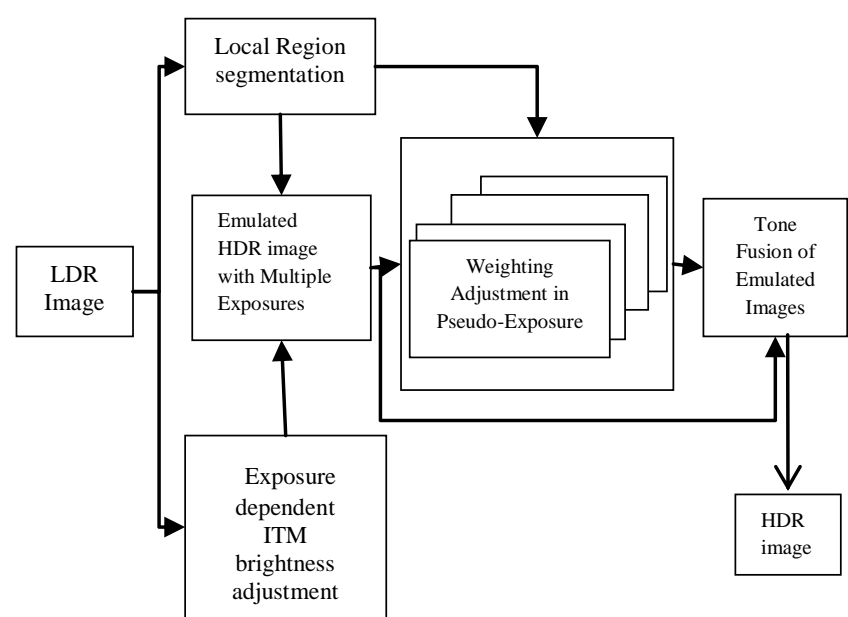

Fig. 1. Tone fusion framework on emulated images with multiple exposures

The techniques used in region-based enhancement include filtering [18], classifying highlights [18], [19], expand map [21], and hallucination [22]. Meylan et al.'s method detects diffuse and specular parts of the image, and expands more on the specular region with a piecewise linear function [19], [20]. Their method is designed mainly for improving highlights on HDR monitors. The PMEBTF method provides the boost detail in both the bright and dark regions so the resulting images would look better. It presents an exposure dependent curve to generate pseudo-multiple-exposures HDR images from a single LDR image. Then it adopts region specific enhancement in pseudo-exposures to boost details in the most distinct regions. In PMEBTF method, the maximum and minimum luminance values are kept in a specific range so the influence of extreme values (maximum and minimum) can be reduced. Experimental results show that the proposed local tone-mapping algorithm can improve the visual quality of the tone-mapped image, especially attention-salient regions. This paper is organized as follows. Section II contains both the saliency-aware weighting and the edge-aware weighting. The proposed weighting is applied in Section III to design a local mapping algorithm for HDR images. Experimental results are presented in Section IV to verify the efficiency of the proposed tone-mapping algorithm. Finally, concluding remarks are provided in Section V.

\section{CONTENT-AWARE WEIGHTING}

In this section, the saliency model in [9] and [10] is first extended to build up a saliency-aware weighting for an HDR image $\left[\Gamma_{b}\left(p^{\prime}\right)\right]$. An edge-aware weighting $\Gamma_{e}\left(p^{\prime}\right)$ is then used for an HDR image by using the local variance and the local mean value of the HDR image. The content aware weighting is then computed as

$$
W\left(p^{\prime}\right)=\frac{\Gamma_{e}\left(p^{\prime}\right)}{\Gamma_{b}\left(p^{\prime}\right)}
$$

\subsection{Saliency-Aware Weighting}

Let $I_{h}\left(p^{\prime}\right)=\left[R_{h}\left(p^{\prime}\right), G_{h}\left(p^{\prime}\right), B_{h}\left(p^{\prime}\right)\right]$ be a pixel in an HDR image. Similar to the saliency model in [9] and [10], our saliency model for the HDR image is based on the image cooccurrence histogram (ICH) of the HDR image. Since each color component of the HDR image is usually represented by floating numbers, it is first quantized such that it is represented by integers. For simplicity, the total number of bins is denoted as $K$, and the color component $R h$ is taken as an example to illustrate the process. The quantized color component is denoted as $Q\left(R_{h}\left(p^{\prime}\right)\right)$. The ICH of the color component $R_{h}$ in intensity domain $H_{i, R}(m, n)$, which represents the occurrence/ co-occurrence of pixel intensity, is defined as

$$
H_{i, R}=\left[H_{i, R}(m, n)\right] ; 1 \leq m, n \leq K
$$

where $H_{i, R}$ is a symmetric square matrix of size $K \times K$. Let $\Omega \rho 1\left(p{ }^{\prime}\right)$ be a square window centered at the pixel $p^{\prime}$ of a radius $\rho 1$. The value of $\rho 1$ is selected as 4 in this paper. An ICH element $H_{i, R}(m, n)$ is the co-occurrence count of image values $m$ and $n$ within the window $\Omega \rho 1\left(p^{\prime}\right)$. A probability mass function (PMF) $P_{i, R}$ is then obtained by normalizing the ICH matrix $H_{i, R}$. Since saliency is usually negatively correlated with occurrence/co-occurrence, an inverted PMF $\bar{P}_{i, R}(m, n)$ is computed as

$$
\left\{\begin{array}{cc}
0 ; & \text { if } P_{i, R}(m, n)=0 \\
0 ; & \text { if } P_{i, R}(m, n)>\frac{1}{U_{i, R}} \\
\frac{1}{U_{i, R}}-P_{i, R}(m, n) ; & \text { if } P_{i, R}(m, n) \leq \frac{1}{U_{i, R}}
\end{array}\right.
$$

where $P_{i, R}(m, n)$ is an element of $P_{i, R}$ and $U_{i, R}$ is the total number of nonzero items in $H_{i, R}$. Visual saliency is computed from the inverted PMF. For a pixel $p^{\prime}$, visual saliency $S_{i, R}\left(p^{\prime}\right)$ is defined as follows:

$$
S_{i, R}\left(p^{\prime}\right)=\sum_{p \in \Omega_{\rho 1}\left(p^{\prime}\right)} \bar{p}_{i, R}\left(Q\left(R_{h}\left(p^{\prime}\right)\right), Q\left(R_{h}(p)\right)\right)
$$

Similarly, $S_{i, G}\left(p^{\prime}\right)$ and $S_{i, B}\left(p^{\prime}\right)$ are computed for the color component $G_{h}$ and the color component $B_{h}$. All of them are normalized to $[0,1]$ and added together to form the saliency model $S_{i}\left(p^{\prime}\right)$ in intensity domain. Besides the saliency computed in intensity domain, gradient orientation is also incorporated in the saliency computation. For each pixel, the gradient orientation is calculated and quantized to 180 bins. An orientation co-occurrence histogram $H_{O, R}$ can be constructed from the quantized gradient orientation. The corresponding inverted PMF $\bar{P}_{o, R}$ and the saliency model $S_{o, R}\left(p^{\prime}\right)$ can then be generated from $H_{o, R}$. Similarly, $S_{o, G}\left(p^{\prime}\right)$ and $S_{o, B}\left(p^{\prime}\right)$ are computed. All of them are normalized to [0, 1] and added together to form the saliency model $S_{o}\left(p^{\prime}\right)$ in gradient domain. The sum of $S_{i}\left(p^{\prime}\right)$ and $S_{o}\left(p^{\prime}\right)$ is denoted as $S\left(p^{\prime}\right)$. The final visual saliency is defined as the normalized value of $S\left(p^{\prime}\right)$

$$
S_{F}\left(p^{\prime}\right)=\frac{1}{N} \sum_{p=1}^{N} \frac{S\left(p^{\prime}\right)}{S(p)}
$$

where $N$ is the total number of pixels in an image. The proposed saliency-aware weighting $\Gamma_{b}\left(p^{\prime}\right)$ is then defined as

$$
\Gamma_{b}\left(p^{\prime}\right)=\left\{\begin{array}{lr}
1 ; & \text { if } S_{F}\left(p^{\prime}\right) \leq 1 \\
S_{F}^{k}\left(p^{\prime}\right) ; & \text { otherwise }
\end{array}\right.
$$

where $\kappa$ (typically between 0.5 and 1 ) is a constant and its value is selected as 0.75 in this paper. The processing priority of the pixel $p$ ' is assigned according to the value of $\Gamma_{b}\left(p^{\prime}\right)$. A higher priority is assigned to a pixel with a larger $\Gamma_{b}\left(p^{\prime}\right)$. The value of $\Gamma_{b}\left(p^{\prime}\right)$ is usually larger than 1 if the pixel $p$ ' is in an attention-salient region. Therefore, a higher priority is usually given to a pixel in an attention-salient region. This matches 
the feature of our HVS, i.e., our HVS pays more attention to the information in attention-salient regions than that outside the attention-salient regions [7].

\subsection{Edge-Aware Weighting}

The luminance value of the HDR image is first obtained by computing a linear combination of the red, green, and blue components as follows:

$$
Y_{h}(p)=0.299 R_{h}(p)+0.587 G_{h}(p)+0.114 B_{h}(p)
$$

The luminance component in log domain is denoted as $L_{h}(p)$ Let $\sigma_{L h, \rho 2}^{2}(p)$ and $\mu_{L h, \rho 2}(p)$ be the variance and the mean value of the component $L_{h}$ in the window $\Omega_{\rho 2}(p)$. The value of $\rho_{2}$ is selected as 15 in this paper. Consider two pixels $p$ and $p^{\prime}$. If $L_{h}(p)$ is at an edge and $L_{h}\left(p^{\prime}\right)$ is in a flat area, the value of $\sigma_{L h, \rho 2}^{2}(p) / \mu_{L h, \rho 2}^{2}(p)$ is usually larger than that of $\sigma_{L h, \rho 2}^{2}\left(p^{\prime}\right) / \mu_{L h, \rho 2}^{2}\left(p^{\prime}\right)$ Based on this observation, an edge aware weighting $\Gamma_{e}\left(p^{\prime}\right)$ is computed by using normalized local variances of all pixels, i.e.,

$$
\Gamma_{e}\left(p^{\prime}\right)=\frac{1}{N} \sum_{p=1}^{N} \frac{\left(\frac{\sigma_{L h, \rho 2}^{2}\left(p^{\prime}\right)+v 1}{\mu_{L h, \rho 2}^{2}\left(p^{\prime}\right)+v 2}\right)^{\zeta}}{\left(\frac{\sigma_{L h, \rho 2}^{2}\left(p^{\prime}\right)+v 1}{\mu_{L h, \rho 2}^{2}\left(p^{\prime}\right)+v 2}\right)^{\zeta}}
$$

where $\zeta$ (typically between 0.5 and 1 ) is a constant and its value is selected as 0.75 . The constant $v 1$ is included to avoid instability when $\sigma_{L h, \rho 2}^{2}\left(p^{\prime}\right)$ is close to zero, and its value is computed as $(0.001 * L) 2$, with $L$ being the dynamic range of the input image [21]. $v 2$ is a small constant (typically 10-9) that prevents division by zero. The value of $\Gamma_{e}\left(p^{\prime}\right)$ is usually larger than 1 if $p^{\prime}$ is at an edge and smaller than 1 if $p^{\prime}$ in a smooth area. Clearly, larger weighting is assigned to a pixel at an edge than a pixel in a flat area by using the proposed weighting $\Gamma_{e}\left(p^{\prime}\right)$ in (4).

\section{SALIENCY-AWARE LOCAL TONE MAPPING}

Psychophysical studies indicate the following: 1) Our HVS appears to select attention-salient regions before further processing so as to reduce the complexity of the scene analysis, and 2) our HVS is not blind to the information outside the attention-salient regions [7]. Therefore, it makes sense to allow distortions according to the visual saliency model to take place after the dynamic range of the HDR image is scaled down. Given an HDR image, the luminance component of the HDR image in (3) is decomposed as

$$
Y_{h}(p)=Y_{b}(p) Y_{d}(p)
$$

where $Y_{b}$ and $Y_{d}$ are the base layer and the detail layer, respectively. The dynamic range of the detail layer $Y_{d}$ is small, while the base layer $Y_{b}$ could vary a great deal, often much more than the detail layer $Y_{d}$. The overall dynamic range is reduced by using a global tone-mapping algorithm to scale down the base layer $\hat{Y}_{b}$ as $\hat{Y}_{b}$. The detail layer $Y_{d}$ is preserved or even amplified as $\hat{Y}_{d}$ to enhance local contrasts. The compressed luminance value $\mathrm{Yl}$ is the product of $\hat{Y}_{b}$ and $\hat{Y}_{d}$. The HDR image $\{R, G, B\}_{h}, Y_{h}$, and $Y_{l}$ are finally adopted to generate the output LDR image $\{R, G, B\}_{l}$. As shown by the "just noticeable difference" experiment [22], the log function approximates the transformation performed by the retina of the HVS. Inspired by this fact, the decomposition is performed in the log domain as

$$
Y_{h}(p)=Y_{b}(p)+Y_{d}(p)
$$

where $L_{b}(p)$ and $L_{d}(p)$ are $\log \left(Y_{b}(p)\right)$ and $\log \left(Y_{d}(p)\right)$ respectively. The three major components of the proposed local tone mapping algorithm are the following: 1) the decomposition of $L_{h}$ into $L_{b}$ and $L_{d} ; 2$ ) the compression of $L_{b}$ via a global tone mapping; and 3) the amplification of $L_{d}$.

\subsection{Decomposition of $L_{h}$ by a Perceptually Guided Filter}

Inspired by the guided filter in [6], $L_{b}$ is assumed to be a linear transform of $L_{h}$ in the window $\Omega_{\rho_{2}}\left(p^{\prime}\right)$

$$
L_{b}(p)=a_{p^{\prime}}(p) L_{h}(p)+b_{p^{\prime}}, \forall p \in \Omega_{\rho 2}\left(p^{\prime}\right)
$$

where $a_{p^{\prime}}$ and $b_{p^{\prime}}$ are supposed to be constant in the window $\Omega_{\rho 2}\left(p^{\prime}\right)$. The linear coefficients $\left(a_{p}, b_{p^{\prime}}\right)$ are obtained by minimizing the difference between $L_{h}$ and $L_{b}$ while maintaining the linear model (7), i.e., by minimizing the following cost function:

$$
\sum_{p \epsilon \Omega_{\rho 2}\left(p^{\prime}\right)} W\left(p^{\prime}\right)\left(a_{p^{\prime}} L_{h}(p)+b_{p^{\prime}}-L_{h}(p)\right)^{2}+\lambda a_{p^{\prime}}^{2}
$$

By comparing the cost function $E\left(a_{p}, b_{p^{\prime}}\right)$ in (8) with the cost function in [15], it can be found that the cost function $E\left(a_{p^{\prime}}, b_{p^{\prime}}\right)$ in (8) contains a content-aware weighting $M(p)$ which is responsible for reducing the halo artifact and preserving fine details according to the saliency-aware weighting. The value of $\Gamma_{e}\left(p^{\prime}\right)$ is usually larger than 1 if $p^{\prime}$ is at an edge and smaller than 1 if $p$ ' is in a flat area. This implies the following: 1) the fidelity of $L_{b}$ with respect to $L_{h}$ is required to be higher by the proposed filter if $p$ ' is at an edge, and 2) the base layer $L_{b}$ is required to be smoother by the proposed filter if $p$, is in a flat area. Therefore, the proposed filter has a potential to preserve sharp edges and smooth flat regions better than the guided filter in [6]. In addition, the proposed filter can smooth attention salient regions better than the guided filter in [6]. As a result, more fine details in the attention-salient regions are preserved in the tone-mapped image via the proposed filter. It is shown in the linear model (7) that $\nabla L_{b}(p)=a_{p} \nabla L_{h}(p)$. Clearly, the smoothness of $L_{b}$ in $\Omega_{\rho 2}\left(p^{\prime}\right)$ depends on the value of $a_{p^{\prime}}$. This implies that the data terms and the regularization terms in (8) are similar to that in [5] in the sense that the data term measures the fidelity of $L_{b}$ with respect to the input $L_{h}$ and the regularization term is on the smoothness of $L b$. The major difference is that the proposed filter and the filter in [15] are based on local optimization while the optimization problem in [13] is global. The optimal values of $a_{p}$, and $b_{p}$, are computed as

$$
\begin{gathered}
a_{p^{\prime}}=\frac{W\left(p^{\prime}\right) \sigma_{L h, \rho 2}^{2}\left(p^{\prime}\right)}{W\left(p^{\prime}\right) \sigma_{L h, \rho 2}^{2}(p)+\lambda} \\
b_{p^{\prime}}=\left(1-\alpha_{p^{\prime}}\right) \mu L_{h, \rho 2}\left(p^{\prime}\right)
\end{gathered}
$$

It is worth noting that a pixel $\mathrm{p}$ is involved in all overlapping windows $\Omega_{\rho 2}\left(p^{\prime}\right)$ 's. There are many different values of $L_{b}(p)$. A simple method is to average all of the possible values of $L_{b}(p)$ as follows:

$$
L_{h}(p)=\bar{a}_{p} L_{h}(p)+\bar{b}_{p}
$$


where $\bar{a}_{p}$ and $b_{p}$ are computed as

$$
\begin{aligned}
\bar{a}_{p} & =\frac{1}{\left|\Omega_{\rho 2}(p)\right|} \sum_{p^{\prime} \in \Omega_{\rho 2}(p)} a_{p^{\prime}} \\
\bar{b}_{p} & =\frac{1}{\left|\Omega_{\rho 2}(p)\right|} \sum_{p^{\prime} \epsilon \Omega_{\rho 2}(p)} b_{p^{\prime}}
\end{aligned}
$$

\section{PSEUDO-MULTIPLE-EXPOSURE TONE FUSION (PMET)}

Inverse tone mapping operators using multiple images of different exposures have the advantages of a higher dynamic range and perceivable details. For legacy LDR images, it is hard to find multiple images with different exposures [15]. It is an exposure dependent $\mathrm{S}$ curve to convert one normal exposure LDR image into pseudo-multiple-exposures. Perceivable regions are different in the pseudo-exposures. For example, the bright region of the dark images and the dark region of the bright images have more visible details. Therefore, it is a region-based weighting adjustment on the pseudo-exposures to boost details in the most distinct regions. Through different brightness adjustments, emulated HDR images with pseudo-multiple-exposures are then generated [15]. Local region segmentation is performed on the original LDR image. It is adopted the accumulated histogram distribution to divide an image into four regions which are applied to the pseudo-exposures. The tone equalization performed by a Gaussian weighting that gives the highest weighting on the most noticeable region in the pseudoexposures. Therefore, we can boost details in the most distinct region in various exposures. By combining these images, the details in different exposure images become all visible in the final produced HDR image [15]. The function of each individual module is depicted as follows.

\subsection{Exposure Dependent Inverse Tone Mapping Function}

The three common kinds of tone curve for adjusting image brightness for cameras, displays or printers are linear, Gamma $(\gamma)$ and curves. The Gamma curve $\left(I^{\gamma}\right)$ is non-linear and includes two cases with $\gamma<1$ and $\gamma>1$ respectively for an input with normalized luminance between zero and one. The $\gamma<1$ case results in a bright-toned image; the $1>1$ one produces a dark-toned image. The $S$ curve enhances the highlight and shadow regions and increases contrast in the important mid-tone area, which is responsible for perceived contrast. Reinhard et. al. propose a method to generate HDR images, and it has shown that the S-shape function can produce better quality HDR images [16]. As the result of high-contrast, we choose the $S$ curve as a mapping function in our proposed approach. Eq. (14) is an inverse tone mapping function that is modeled as S-shape and is reversible [16], [23], [25].

$L_{w}(i, j)=\left\{\begin{array}{c}\frac{c \cdot L_{a d} \cdot L_{d}(i, j)}{1-L_{d}(i, j)}, \text { if } L_{d}(i, j)<1 \\ L_{\max }=c \cdot L_{a d} \cdot L_{s \max } . \quad L_{d}(i, j)=1\end{array}\right.$

The $L_{d}(i, j)$ and $L_{w}(i, j)$ indicate the luminance of the reference LDR image and the HDR image at pixel $(i, j)$ individually. The $L_{a d}$ is the average luminance value of a HDR image; is a multiplication factor. The $L_{d}(i, j)$ expresses displayable luminance in the range of $0 \sim 1$. Although, $L_{a d}$ represents the geometric average of the world luminance image, we can approximate $L_{a d}$ using the geometric average of the LDR image when the LDR image is not overexposed or under-exposed [24]. $L_{a d}$ is an approximation of the key of the scene. During inverse tone mapping, the can be used to adjust the key of the mapped HDR image.

The $L_{\max }$ can be set based on the maximum luminance of the target HDR image. A piecewise linear function is adopted to approximate the proposed tone curve and to derive the maximum value. In addition, we assume the ratios of the consecutive slopes are the same. From the above assumptions, the $L_{\max }$ is expressed as $c . L_{a d} L_{\text {smax }}$ is an adjustable multiplication factor to set the maximum value. The $L_{\text {smax }}$ is a constant set to be 382.5 in the simulations.

The other is the luminance difference between adjacent pseudo-exposures. To make each pseudo-exposure have similar contribution during fusion, the luminance difference between adjacent pseudo-exposures is designed to be equal. The mapping curve is shown below.

$$
\begin{gathered}
L_{w k}(i, j)= \\
L_{w}(i, j)=\left\{\begin{array}{c}
\frac{10^{-P_{k} L_{a d, k} \cdot L_{d}(i, j)}}{1-L_{d}(i, j)}, \text { if } L_{d}(i, j)<1 \\
L_{\text {max }, k=}=10^{-P_{k}} \cdot L_{a d} \cdot L_{\text {smax }} . \text { otherwise }
\end{array}\right.
\end{gathered}
$$

$L_{w k}$ is the world luminance of the pseudo-exposure HDR image. $P_{k}$ is a scaling factor of the th image and is used to control the luminance difference between the adjacent pseudoexposures. The log-average luminance is a useful approximation to the key of the scene [23]. We adopt $10^{P_{k}}$ to control the luminance difference. When $P_{k}$ increases, the luminance difference between adjacent images increases. $L_{a d, k}$ controls the average luminance of the th pseudoexposure image. An adaptive function to adjust the average luminance $L_{a d, k}$ is presented in Eq. (16) [25].

$$
L_{a d, k}=1+\exp \left(\mu E V_{k}\right)
$$

Where $E V_{k}$ indicates the exposure value of the Kth image and is an adjustable constant. In our works, we set equal to 0.85 for simplification. An exponential function is used for a fine control of the average luminance. The larger the $E V_{k}$ is, the higher the average luminance of the produced image is. The maximum mapped HDR luminance is $L_{\max }$, which is controlled by $P_{k}$, and. This is designed based on the dynamic range in the application. In our design, the $L_{\text {smax }}$ is set to a constant. After performing selective local-region enhancement on emulated images, the next step is to fuse these five images into the final HDR image.

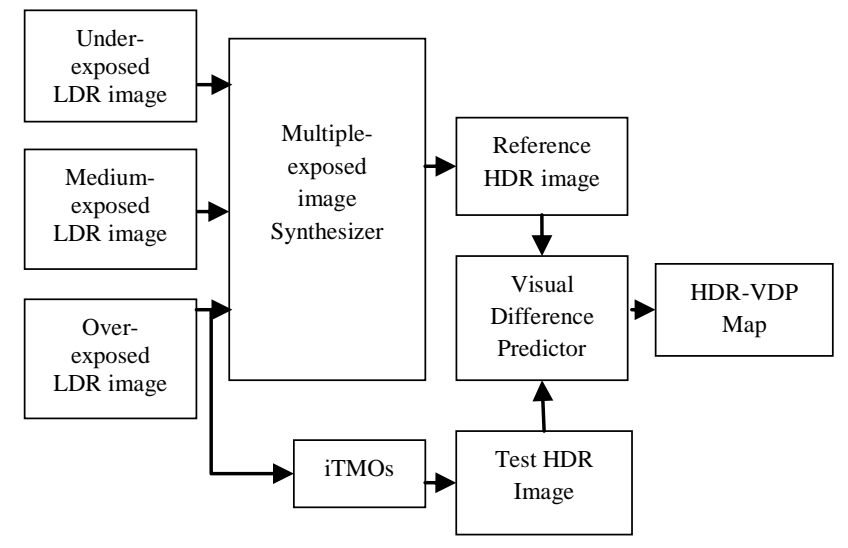

Fig. 2.Block Diagram of PMEBTF Method [15] 
To retrieve the details in highlight and shadow regions and to increase the dynamic range, the final reconstructed HDR image is produced by the weighted average luminance from multiple images as shown in Eq. (17)

$$
L_{w-\operatorname{comb}(i, j)}=\sum_{k=1}^{L} w k(\mathrm{i}, \mathrm{j}) . L_{w k(i, j)} \backslash \sum_{k=1}^{L} w k(i, j)
$$

Where $L_{w-\operatorname{comb}(i, j)}$ represents the luminance of the reconstructed HDR image, $w k_{(i, j)}$. is the weighting value for the Kth image and $\mathrm{L}$ is the number of images. After tone fusion of the emulated images, we convert the luminance back to the color domain through the color correction function.

\section{EXPERIMENTAL RESULTS}

Tone mapping of HDR images is a very hot research topic in

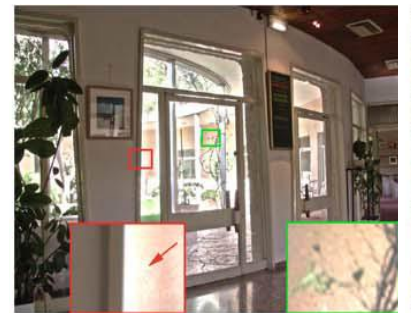

(a)

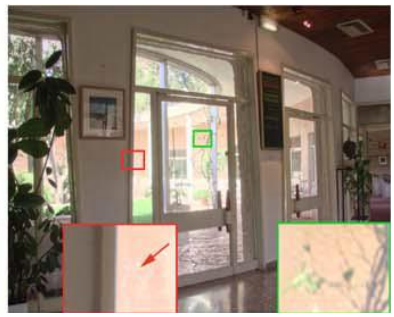

(b)

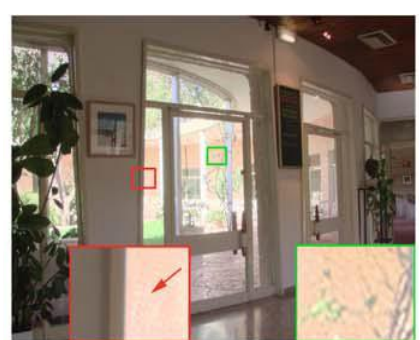

(c)

Fig. 3. Comparison tone-mapping algorithms (a) Original image (b) Tone-mapped images by a local tone-mapping algorithm with the saliency-aware weighting (c) Tonemapped images by PMEBTF Method.

the fields of image processing and computation photography; there are dozens of tone-mapping algorithms. Since this paper focuses on incorporating a saliency with edge-aware weighting (SEAW) into tone mapping of HDR images, and the PMEBTF algorithms are compared. As a result, the SEAW tone-mapping algorithm can avoid halo artifacts from appearing in the tone-mapped images. While the PMEBTF algorithm gives better visual quality as compared to the saliency-aware weighting method of tone-mapped images.

\section{CONCLUSION AND DISCUSSION}

The saliency with edge-aware weighting (SEAW) tone mapping method for HDR images demonstration gives suitable display. Experimental results show that halo artifacts could be avoided from appearing in the tone-mapped image and the visual quality of the tone-mapped image, especially the attention-salient regions, is improved. While the PMEBTF algorithms with tone fusion method to generate one HDR image from a single LDR.

\section{REFERENCES}

[1] P. E. Debevec and J. Malik, Aug. 1997, "Rendering high dynamic range radiance maps from photographs," in Proc. SIGGRAPH, Los Angeles, CA, USA.
[2] J. Li, Y. H. Tian, T. J. Huang, and W. Gao, Nov. 2010, "Probabilistic multi-task learning for visual saliency estimation in video," Int. J. Comput. Vis., vol. 90, no. 2, pp. 150-165.

[3] E. Reinhard, G. Ward, S. Pattanaik, and P. E. Debevec, 2005, High Dynamic Range Imaging: Acquisition, Display and Image-Based Lighting. San Mateo, CA, USA: Morgan Kaufmann.

[4] R. Fattal, D. Lischinski, and M.Werman, Jul. 2002 , "Gradient domain high dynamic range compression," ACMTrans. Graphics, vol. 27, no. 3, pp. 67:1-67:10.

[5] Z. Farbman, R. Fattal, D. Lischinski, and R. Szeliski, Aug. 2008, "Edge-preserving decompositions for multiscale tone and detail manipulation," ACM Trans. Graphics, vol. 21, no. 3, pp. 249-256.

[6] K. He, J. Sun, and X. Tang, Jun. 2013, "Guided image filtering," IEEE Trans. Pattern Anal. Mach. Learn., vol. 35, no. 6, pp. 1397-1409.

[7] L. Itti, C. Koch, and E. Niebur, Nov. 1998 , "Amodel of saliency-based visual attention for rapid scene analysis," IEEE Trans. Pattern Anal. Mach. Intell., vol. 20, no. 11, pp. 1254-1259.

[8] F. Perazzi, P. Krahenbuhl, Y. Pritch, and A. Hornung, Jun. 2012 , "Saliency filters: Contrast based filtering for salient region detection," in Proc. IEEE Int. Conf. Comput. Vis., Providence, RI, USA, pp. 733-740.

[9] S. Lu and J. H. Lim, Oct. 2012, "Saliency modeling from image histograms," in Proc. 12th Eur. Conf. Comput. Vis., Florence, Italy, pp. 321-332.

[10] S. Lu, C. Tan, and J. H. Lim, Jan. 2014, "Robust and efficient saliency modeling from image co-occurrence histograms," IEEE Trans. Pattern Anal. Mach. Learn., vol. 36, no. 1, pp. 195-201.

[11] R. Shen, I. Cheng, and A. Basu, Jun. 2013, "QoE-based multi-exposure fusion in hierarchical multivariate Gaussian CRF," IEEE Trans. Image Process., vol. 22, no. 6, pp. 2469-2478.

[12] E. Reinhard, Nov. 2003, "Parameter estimation for photographic tone reproduction," J. Graphics Tools, vol. 7 , no. 1 , pp. $45-51$.

[13] J. Kuang, H. Yamaguchi, C. Liu, G. M. Johnson, and M. D. Fairchild, Jul. 2007, "Evaluating HDR rendering algorithms," ACM Trans. Appl. Perception, vol. 2, no. 4, pp. 9:1-9:27.

[14] Zhengguo Li and Jinghong Zheng, Dec. 2014, "VisualSalience-Based Tone Mapping for High Dynamic Range Images" IEEE Transactions On Industrial Electronics, Vol. 61, No. 12.

[15] Tsun-Hsien Wang, Cheng-Wen Chiu, Wei-Chen Wu, Jen-Wen Wang, Chun-Yi Lin, Ching-Te Chiu, and JingJia Liou, April 2015, "Pseudo-Multiple-Exposure-Based Tone Fusion with Local Region Adjustment" IEEE Transactions On Multimedia, Vol. 17, No. 4.

[16] E. Reinhard, T. Kunkel, Y. Marion, J. Brouillat, R. Cozot, and K. Bouatouch, Dec. 2007, "Image display algorithms for high and low dynamic range display devices,” J. Soc. Inf. Display, vol. 15, no. 12, pp. $997-$ 1014. 
[17] F. Banterle, A. Artusi, K. Debattista, and A. Chalmers, 2011, "Advanced High Dynamic Range Imaging: Theory and Practic". Natick, MA, USA.

[18] P. Didyk, R. Mantiuk, M. Hein, and H.-P. Seidel, 2008, "Enhancement of bright video features for HDR displays," in Comput. Graphics Forum (Proc. Eurograph. Symp. Rendering 2008 Sarajevo, Bosnia, Herzegovina), vol. 27 , no. 4, pp. 1265-1274.

[19] L. Meylan, S. Daly, and S. Süsstrunk, 2006, "The reproduction of specular highlights on high dynamic range displays," in Proc. IS\&T/SID 14th Color Imaging Conf., 2006, pp. 333-338.

[20] L. Meylan, S. Daly, and S. Susstrunk, 2007, "Tone mapping for high dynamic range displays," in Proc. IS\&T/SPIE Electron. Imaging: Human Vis. Electron. Imaging XII, vol. 6492, pp. 649210-1-649210-12.
[21] F. Banterle, P. Ledda, K. Debattista, and A. Chalmers, 2006, "Inverse tone mapping," in Proc. GRAPHITE, pp. 349-356.

[22] L. Wang, L.-Y. Wei, K. Zhou, B. Guo, and H.-Y. Shum, 2007, "High dynamic range image hallucination," in Proc. ACM SIGGRAPH 2007.

[23] E. Reinhard, M. Stark, P. Shirley, and J. Ferwerda, 2002, "Photographic tone reproduction for digital images," ACM Trans. Graph., vol. 21, no. 3, pp. 267-276.

[24] K. K. Biswas and S. N. Pattanaik, 2005, "A simple spatial tone mapping operator for high dynamic range images operator for high dynamic range images," in Proc. IS\&T/SID 13th Color Imaging Conf., pp. 291-296.

[25] G. Messina, A. Catorina, S. Battiato, and A. Bosco, Jul. 2003, "Image quality improvement by adaptive exposure correction techniques," in Proc. IEEE Int. Conf. Multimedia Expo, vol. 1, pp. I-549-I-552. 